\title{
MEK Pathway Inhibition Increases the Efficacy of a PI3K and HDAC Inhibitor in Endometrial Cancer Cells
}

Konstantinos Polymeros ( $\square$ kpolymeros@doctors.org.uk)

University of Leicester

David S. Guttery

University of Leicester

Salvador Macip

University of Leicester

Esther L. Moss

University of Leicester

\section{Research Article}

Keywords: Endometrial, cancer, CUDC-907, MEK, PI3K, PD0325901

Posted Date: October 13th, 2021

DOl: https://doi.org/10.21203/rs.3.rs-575548/v1

License: (c) (i) This work is licensed under a Creative Commons Attribution 4.0 International License. Read Full License 


\section{Abstract}

Background: Endometrial cancer (EC) is the commonest gynaecologic malignancy in many countries and its incidence is rising. Although excellent prognosis is associated with early stage disease, response to systemic treatment for metastatic or recurrent EC is often low and treatment options are limited.

Aims-Methods: The aim of the study was to propose improved targeted drug treatments suitable for subsequent testing in pre-clinical models of EC. Cell proliferation assay (MTS) was used to assess viability of EC cell lines following treatment with drug inhibitors and Western blotting to explore the effect of inhibitors in molecular pathways.

Results: We identified that CUDC-907, a PI3K and HDAC inhibitor, was the most effective monotherapy treatment of a panel of drugs screened in EC cells. Moreover, several combination treatments showed synergism in EC cell lines, with the most efficacious being CUDC-907 combined with the MEK inhibitor PD0325901. This indicates that simultaneous inhibition of two main oncogenic pathways, PI3K and MEK, could improve drug sensitivity in EC.

Conclusions: In summary, we propose a range of targeted inhibitory drugs, alone or in combination, showing in vitro efficacy in endometrial cancer cells, which could provide novel therapeutic strategies for advanced EC.

\section{Background}

The incidence of endometrial cancer (EC) is rising in many countries, with an estimated of 417,367 new cases and 97,370 deaths worldwide in 2020 (1). Unlike many other cancers, where the prognosis has improved over time, mortality from EC is rising in many countries, including the UK (2). In the USA, endometrial and cervical cancer are the only malignancies for which the five-year survival has decreased when comparing the 1975-77 period with 2006-12 (from 86.9-83.4\% for EC) (3). At present the first-line systemic therapy for advanced EC is platinum-based chemotherapy, however response rates are modest. A Cochrane review (4) identified three main combinations: Cisplatin with doxorubicin, cisplatin with doxorubicin and paclitaxel and cisplatin with doxorubicin and cyclophosphamide and although adjuvant chemotherapy following surgery did improve overall survival there is still uncertainty as to the optimum regimen. New agents, in particular immune checkpoint inhibitors, appear to hold great potential for EC cases with microsatellite instability $(\mathrm{MSH}-\mathrm{H})$ /mis-match repair gene deficiencies (dMMR), either as monotherapy or in combination with other agents, for example a multireceptor tyrosine kinase inhibitor, however outcome in microsatellite stable (MSS) tumours is lower (5).

EC is reported to contain the greatest proportion of PI3K mutations of all cancers (6). Of these, PTEN, PIK3CA and PIK3R1 are the most commonly mutated, with rates of $63.5 \%, 52.2 \%$ and $30.9 \%$ respectively, much higher to any other cancer studied in The Cancer Genome Atlas dataset (7). It is therefore unsurprising that many novel targeted therapies for recurrent/advanced EC focus on this pathway. 
$\mathrm{PI} 3 \mathrm{~K}$ is a family of kinases that are categorised into three classes. Class I is the best described and most involved in tumourigenesis. The Class I catalytic subunit (p110a) is encoded by the PIK3CA gene, while its regulatory subunit (p85) is encoded by the PIK3R1-3 genes (8). Signals from tyrosine kinase receptors (RTK) and G-protein-coupled receptors in the cell membrane lead to PI3K activation, which in turn phosphorylates phosphatidylinositol 4,5-bisphosphate (PIP2) and converting it to phosphatidylinositol 3,4,5-triphosphate (PIP3). PIP3 can be dephosphorylated by the PTEN phosphatase and reverted back to PIP2, which halts the downstream effects of PIP3. In the absence of this action, PIP3 recruits phosphoinositide-dependent kinase-1 (PDK1) and protein kinase B (AKT) in the inner cell membrane. PDK1 subsequently phosphorylates AKT and this activity increases even further following AKT's phosphorylation by the mammalian target of rapamycin complex 2 (mTORC2) (9).

The activated AKT has several downstream effectors (10). One of the most important is the activation of the mammalian target of rapamycin complex 1 (mTORC1), which results in increased synthesis of proteins and survival, common feature of most cancers (11).

Another well-studied intracellular signalling pathway is the MAPK, which is responsible for several key cellular functions, including proliferation, differentiation and apoptosis (12), and is commonly involved in many cancers (13). Studies have also proposed a direct association between oestrogenic stimulation (common feature in the majority of endometrial cancers) and MAPK activation (14). Each MAPK pathway is a phosphorylating cascade that involves a minimum of three kinases (MAP3K, MAP2K and MAPK) (15). Five MAPK cascades have been characterized in mammals (16) with the most extensively investigated being the extracellular signal-regulated kinases 1 and 2 (ERK1 and ERK2). The MAPK pathway is triggered by cell surface RTK stimulation, followed by Ras activation (16). Ras will then recruit Raf and MEK1/2 phosphorylation subsequently ensues, followed by ERK1/2 phosphorylation.

Several PI3K and/or mTOR inhibitors have been tested in vitro with encouraging results (17-19), however, to date have failed to demonstrate efficacy in clinical trials when tested as monotherapies (20-23).

Similar outcomes have been reported with MEK inhibitors, with in vitro efficacy demonstrated with several agents (Cobimetinib, U0126, Sorafenib and Selumetinib) (24-27) but failure in early phase clinical trials $(28,29)$. This therefore raises the question as to whether combination therapy, rather than monotherapy, would be a better strategy for any of these targeted agents in clinical practice, as well as highlighting the need for personalized therapies matching targeted inhibitors to driver mutations within the tumour.

To explore novel potential therapies for $\mathrm{EC}$, we tested a PI3K and histone deacetylase (HDAC) inhibitor (CUDC-907) together with a panel of MEK inhibitors, aiming to identify synergistic combinations for killing endometrial cancer cells carrying different driver mutations.

\section{Methods}

Cell culture. The Ishikawa cancer cell line was obtained from Merck KGaA (Darmstadt, Germany) and the HEC-1-A cell line from American Type Culture Collection (ATCC ${ }^{8}$ HTB-112 ${ }^{\text {TM }}$, Manassas, Virginia, USA). Ishikawa cells were cultured in Dulbecco's Modified Eagle's Medium (DMEM), supplemented with $5 \%$ Fetal 
Bovine Serum (FBS) and 1\% Non Essential Amino Acids (GibcoTM). HEC-1-A cells were cultured in McCoy's 5A (Modified) medium (GibcoTM), supplemented with 10\% FBS. All cells were incubated at 37oC in $5 \% \mathrm{CO} 2$.

Cell proliferation assay. The CellTiter $96 \AA$ AQueous One Solution Cell Proliferation Assay (MTS assay, PromegaTM) was used to assess cell viability following treatment with various inhibitory drugs. 5,00010,000 cells were transferred to 96 -well, flat bottom plates, in triplicates. When reached $70-80 \%$ confluence, they were treated with serial concentrations of various inhibitors. Three control wells were used by adding DMSO to it, as all the drugs of our study were diluted in DMSO. Finally, three wells were filled with culture medium only in order to measure the background absorbance. 48-hours after the drug treatment, the reagent was added to the cells at a concentration of 5:1. Following a 2-3 hours incubation in a humidified, $5 \%$ CO2 atmosphere, the absorbance of the plate was read at 490nm using Infinite ${ }^{\circledR}$ F50 / Robotic ELISA plate reader (TECANTM) and the data recorded with the MagellanTM data analysis software. The percentage of cell viability was subsequently calculated with Microsoft Excel 2013 by dividing the absorbance (optical density) of the sample with the one of the control and multiplying this by 100. Graphs were created using GraphPad Prism 7.0 software.

Immunoblotting. After treating cells with drug inhibitors, their pellets were collected and lysed with RIPA lysis buffer containing $150 \mathrm{mM} \mathrm{NaCl}, 50 \mathrm{mM}$ Tris HCl pH 8.0, 1\% Tergitol-type NP-40, 0.1\% Sodium Dodecyl Sulfate, $0.5 \%$ sodium deoxycholate diluted in distilled water, along with $1 \%$ protease inhibitor and $1 \%$ phosphatase inhibitor cocktails (Sigma-AldrichTM). Following centrifugation, the supernatants were collected and the protein concentration was calculated using the Bradford assay. Polyacrylamide gel electrophoresis was subsequently performed and the proteins from the gel were transferred to nitrocellulose filter membrane (Amersham; GE). The membranes were incubated with primary antibodies overnight. They were subsequently washed and incubated with secondary antibodies [Goat anti-Rabbit for rabbit primary antibodies (IRDye $\circledast$ 680RD Goat anti-Rabbit IgG, Li-CORTM) and Goat anti-Mouse for mouse primary antibodies (IRDye ${ }^{\circledR} 800 \mathrm{CW}$ Goat anti-Mouse IgG, Li-CORTM), concentration 1:10,000]. The protein bands analyzed and quantified using Image Studio Lite software version 5.2 (Li-CORTM).

Statistical analysis. IC50 values of inhibitory drugs were calculated after converting the cell viability values derived from MTS assay to percentage of inhibition. This was then converted to a logarithmic value and from the linear regression graph created with GraphPad Prism version 7.0, the IC50 value was calculated. Calculation Index $(\mathrm{Cl})$ for combination drug treatments was calculated using the Chou-Talalay method (30). If $\mathrm{Cl}<1$, there is synergism, if $\mathrm{Cl}>1$ there is antagonism and when $\mathrm{Cl}=1$, there is additive effect. Cl was calculated using CalcuSyn Version 2.0 software (Biosoft).

\section{Results}

\section{EC cancer cells are sensitive to dual PI3K/HDAC inhibition but not to MEK inhibitors.}

In order to screen for potential targeted therapies in EC, two endometrial cancer cell lines (Ishikawa and HEC-1-A) were chosen, due to their different properties and genetic background: Ishikawa is known to 
carry PTEN and PIK3R1 mutations (18), while HEC-1-A carries KRAS and PIK3CA mutations. Moreover, Ishikawa was derived from a well differentiated uterine adenocarcinoma of an Asian patient, while HEC-1A was derived from a moderately differentiated papillary adenocarcinoma of a Caucasian background. We first tested the sensitivity of the cell lines to different targeted monotherapies. Starting with CUDC-907, a novel PI3K and HDAC inhibitor shown to be effective in stopping the proliferation of malignant B cells (31). Both cell lines showed sensitivity to CUDC-907 at low concentrations (Figure 1A). As expected, the efficacy was greater in cells carrying PI3K pathway mutations (Ishikawa, IC50=23nM) compared to cells with wild-type PI3K signalling (HEC-1-A, IC50=129nM). We also treated these cells with a panel of MEK inhibitors (Selumetinib, Trametinib and PD0325901, Figure 1A). These inhibitors did not induce cell death in Ishikawa when used as monotherapy. However, in HEC-1-A cells, reduced viability was observed, consistent with a dependence on an activated RAS-MEK-ERK pathway, but only at the higher concentrations tested (over $1 \mu \mathrm{M}$ ). Of note, HEC-1-A cells had different sensitivity to each MEK inhibitor used, with PD0325901 being the most effective and Selumetinib having no significant effect.

To better understand the effect of these drugs in EC cells, we analysed how they changed the activation of the PI3K and MAPK pathways. As expected, CUDC-907 reduced the basal levels of AKT phosphorylation in Ishikawa (Figure 1B). On the other hand, none of the MEK inhibitors blocked activation of the MAPK pathway in Ishikawa and instead, MAPK and MEK phosphorylation was increased (Figure 1C). In contrast, a high concentration of PD0325901 reduced MAPK phosphorylation in HEC-1-A cells (Figure 1C). This shows that the toxicity of the MEK inhibitors is not proportional to their inhibition of the pathway. Our results together confirm the relevance of choosing targeted inhibitors based on the pathways mutated in each tumour. They also confirm that inhibiting the PI3K pathway may be in general more useful than inhibiting RAS-MEK-ERK in EC and highlight the varied responses to different MEK inhibitors.

\section{Trametinib has a synergistic effect with CUDC-907 in cells with oncogenic MAPK activation. We next} explored potential synergistic combinations between PI3K and MEK inhibitors. We selected Trametinib and PD0325901, but discarded Selumetinib due to its lack of efficacy in our models. As shown in Figure 2A, adding Trametinib did not change the sensitivity of Ishikawa to CUDC-907, however, the combination had a synergistic effect in HEC-1-A cells, combination index $(\mathrm{Cl})$ of 0.878 , suggesting that PI3K and MEK inhibition together may be a good therapeutic option for EC cells that carry mutations in both pathways. Although Trametinib alone did not suppress the basal levels of phosphorylated MAPK (see Figure 1C), when combined with CUDC-907, it reduced MAPK activity in a dose-dependent manner (Figure 2B). Interestingly, this effect was seen in both cell lines, and therefore independent of the oncogenic activation of the pathway. This indicates that the degree of MAPK inhibition is not proportional to the increase in cell death observed with these combination therapies, suggesting that other off-target effects of these drugs may be contributing to the results.

\section{PD0325901 synergizes with CUDC-907 regardless of the mutation status of the MAPK pathway.}


We also tested the combination of CUDC-907 and PD0325901 in EC cells. As shown in Figure 3A, the treatment had synergistic effects in both cell lines. Of note, this was the only combination of CUDC-907 with a MEK inhibitor that showed strong synergism in the Ishikawa cells, $\mathrm{Cl}$ of 0.381 , as it reduced viability from $66 \%$ (when Ishikawa cells are treated with CUDC $0.01 \mu \mathrm{M}$ alone) to $36 \%$ or $25 \%$ when adding $0.1 \mu \mathrm{M}$ or $1 \mu \mathrm{M}$ of PD0325901, respectively. This suggests that certain MEK inhibitors may be effective even in cells with no mutations in the RAS-MEK-ERK pathway. The synergism was still associated with a strong suppression of the basal MAPK pathway activity in these cells (Figure 3B). These data together show that some MEK inhibitors can enhance the effects of PI3K inhibitors in EC cells, even those with no mutations in the MAPK pathway.

\section{Discussion}

There is a pressing clinical need for more efficacious systematic therapies for endometrial cancer. In this study we have identified the PI3K and HDAC inhibitor CUDC-907 as being active in EC cell lines.

We have identified that CUDC-907, a PI3K and HDAC inhibitor, was very effective against EC cells, with IC50 values in the nanomolar level. CUDC-907 is currently under investigation in early phase trials, primarily in haematological malignancies, but also in solid tumours (32). To our knowledge, it has not been investigated previously in endometrial cancer and since it has a favourable safety profile $(33,34)$, it is an attractive candidate for further testing in pre-clinical models.

Our rationale for investigating combinations with MEK inhibitors was born out of the disappointing results of PI3K and mTOR inhibitors tested as monotherapies in clinical trials, for example everolimus, pilaralisib and others (20-23) but also evidence suggesting that MAPK is activated in EC following oestrogen receptor stimulation $(14,35)$.

Our results suggest that, while monotherapies with MAPK inhibitors may have a limited use in EC, they can successfully enhance other targeted drugs. We have shown that the combination of PI3K and MAPK inhibition can be synergistic and, in some circumstances even independently of the activation status of the pathway. This indicates that phosphorylation of different members of the MAPK pathway would not be sufficient to predict sensitivity to these drugs. In our study we identified the paradoxical activation of the MEK-ERK pathway when cells were treated with MEK inhibitors, as shown by the increased basal levels of MEK and MAPK phosphorylation (Figure 1C). This was also observed previously in a study of our lab made on chronic lymphocytic leukemia, which proposed an upstream reactivating loop as a possible cause (36). This paradoxical activation was more prominent in Ishikawa cells, possibly as a result of wild type genes controlling the MAPK pathway. On the contrary, HEC-1-A cells, which carry KRAS mutations, have a constitutively more active MAPK pathway, and hence this paradoxical activation is more subtle and can be reversed with higher concentrations of MEK inhibitors.

The association between KRAS mutations and MEK inhibitor resistance has been studied previously. In colorectal cancer, a direct correlation between the strength of ERK1/2 signalling and the sensitivity of colorectal cells to MEK1/2 inhibitors (37). This is in keeping with our results, which confirmed that the 
KRAS-mutant HEC-1-A cells are more sensitive to MEK inhibitors than the Ishikawa cells. Similarly, a study on 13 EC cell lines concluded that KRAS-mutant cell lines (including HEC-1-A), were more sensitive to the MEK inhibitor Cobimetinib compared to the PTEN-mutant cell lines (24), again in keeping with our results.

The most efficacious combination treatment was CUDC-907 and PD0325901, a drug shown to be safe in a phase 1 clinical trial (29), with efficacy independent of the mutational status of the MAPK pathway. In contrast, the combination of Trametinib and PI3K inhibitor (CUDC-907) was only synergistic in cells carrying a mutated MEK pathway. Interestingly, the synergism observed with these two drug combinations was irrespective of the degree of MAPK inhibition, as both these MEK inhibitors, when used as monotherapy, do not suppress the MAPK pathway. This might be related to complex and variable offtarget and signaling effects associated with MEK inhibitors (38).

\section{Study limitations.}

The cells lines (HEC-1A and Ishikawa) were chosen because of their different mutational profiles and suited the screening nature of this study. It would arguably be better to perform the experiments in a larger panel of cell lines, which could be more representative of EC, and confirm the prediction of any sensitivity to a certain drug by selectively silencing the appropriate pathway using CRISPR-Cas 9 or other genetic tools. Also, there is a longstanding and ongoing debate as to the value of cell lines as a cancer model, although they do have a proven track record of successes (39). Proponents of cell line work emphasize the fact that it is a cheap and easy to grow screening tool available to almost every laboratory, enabling extensive testing, which would be very challenging and costly to conduct in human or animal models.

A negative of cell lines is that they are unable to capture the tumour microenvironment, which is known to impact on tumour behaviour. In vitro cells are also a very poor model in assessing drug-induced toxicities, as these can only be reliably assessed in in vivo models (40).

\section{Conclusions}

In summary, the novel PI3K and HDAC inhibitor (CUDC-907), was very effective in reducing viability of endometrial cancer cell lines both as monotherapy and in combination with the MEK inhibitor PD0325901. We propose this drug and combination be taken forward for further testing in pre-clinical models with a view to a potential new combination in the systemic treatment of EC.

\section{Abbreviations}

AKT Protein kinase $B$

$\mathrm{Cl}$ Calculation Index

DMEM Dulbecco's Modified Eagle's Medium 
DMSO Dimethyl Sulfoxide

EC Endometrial cancer

ERK Extracellular signal-regulated kinase

FBS Fetal Bovine Serum

HDAC Histone deacetylase

IC50 The concentration of a drug that is required for $50 \%$ inhibition in vitro

MAPK Mitogen-activated protein kinase

MMR Mismatch repair

mTOR mammalian target of rapamycin

PDK Phosphoinositide-dependent kinase

PI3K Phosphoinositide 3-kinase

PIP2 Phosphatidylinositol 4,5-bisphosphate

PIP3 Phosphatidylinositol 3,4,5-triphosphate

RIPA Radio immunoprecipitation assay

RTK Receptor tyrosine kinase

\section{Declarations}

Ethics approval and consent to participate: Not applicable

Consent for publication: Not applicable

Availability of data and materials: All data generated or analysed during this study are included in this published article [and its supplementary information files].

Competing interests: EM serves on advisory boards for Inivata and GlaxoSmithKline; received speaker fees from GlaxoSmithKline; has received research grants from Intuitive Surgical and Hope Against Cancer for unrelated work. All other authors have no competing interests.

Funding: We received a Cancer Research UK (CRUK) development grant and funding from the charity HOPE against cancer. The funding bodies had no role in the design of the study and the analysis and interpretation of data and in writing the manuscript 
Authors' contributions: Experiments were designed and analysed by SM and KP, with DG and EM. All experiments were performed by KP. The manuscript was written by KP, SM and EM. All authors revised and edited the manuscript.

Acknowledgements: This work was supported by the M.C. Andreu Memorial Fund.

\section{References}

1. Sung H, Ferlay J, Siegel RL, Laversanne M, Soerjomataram I, Jemal A, et al. Global cancer statistics 2020: GLOBOCAN estimates of incidence and mortality worldwide for 36 cancers in 185 countries. CA: a cancer journal for clinicians 2021 Feb 4,.

2. Cancer Research UK. 2019.

3. Ahmedin Jemal et al. Annual Report to the Nation on the Status of Cancer, 1975-2014, Featuring Survival. Journal of the National Cancer Institute 2017 March 31;109(9).

4. Galaal K, Al Moundhri M, Bryant A, Lopes AD, Lawrie TA. Adjuvant chemotherapy for advanced endometrial cancer. The Cochrane database of systematic reviews 2014 May 15,(5):CD010681.

5. Makker V, Taylor MH, Aghajanian C, Oaknin A, Mier J, Cohn AL, et al. Lenvatinib Plus Pembrolizumab in Patients With Advanced Endometrial Cancer. Journal of clinical oncology 2020 Sep 10,38(26):2981-2992.

6. TCGA RN. Integrated genomic characterization of endometrial carcinoma. Nature 2013;497:67-73.

7. Cyriac Kandoth, Michael D McLellan, Fabio Vandin, Kai Ye, Beifang Niu, Charles Lu, et al. Mutational landscape and significance across 12 major cancer types. Nature 2013 Oct 17,;502(7471):333-339.

8. Cheng H, Roberts TM, Zhao JJ, Liu P. Targeting the phosphoinositide 3-kinase pathway in cancer. Nature Reviews Drug Discovery 2009 Aug;8(8):627-644.

9. Manning BD, Toker A. AKT/PKB Signaling: Navigating the Network. Cell 2017 Apr 20;169(3):381-405.

10. Vanhaesebroeck B, Alessi DR. The PI3K-PDK1 connection: more than just a road to PKB. The Biochemical journal 2000 Mar 15;;346 Pt 3(3):561-576.

11. Martini M, De Santis MC, Braccini L, Gulluni F, Hirsch E. PI3K/AKT signaling pathway and cancer: an updated review. Annals of Medicine 2014 Sep;46(6):372-383.

12. Morrison DK. MAP kinase pathways. Cold Spring Harbor perspectives in biology 2012 Nov $1 ;$;(11):a011254.

13. Dhillon AS, Hagan S, Rath O, Kolch W. MAP kinase signalling pathways in cancer. Oncogene 2007 May 14;26(22):3279-3290.

14. Zhou L, Cai B, Bao W, He Y, Chen X, Yang Y, et al. Crosstalk Between Estrogen Receptor and MitogenActivated Protein Kinase Signaling in the Development and Progression of Endometrial Cancer. International journal of gynecological cancer 2011 Nov;21(8):1357-1365.

15. Shaul YD, Seger R. The MEK/ERK cascade: From signaling specificity to diverse functions. BBA Molecular Cell Research 2007;1773(8):1213-1226. 
16. Qi M, Elion EA. MAP kinase pathways. Journal of cell science 2005 Aug 15;118(Pt 16):3569-3572.

17. Keiko Shoji, Katsutoshi Oda, Tomoko Kashiyama, Yuji Ikeda, Shunsuke Nakagawa, Kenbun Sone, et al. Genotype-Dependent Efficacy of a Dual PI3K/mTOR Inhibitor, NVP-BEZ235, and an mTOR Inhibitor, RAD001, in Endometrial Carcinomas. PLoS One 2012 May $1,7(5): \mathrm{e} 37431$.

18. Weigelt B, Warne PH, Lambros MB, Reis-Filho JS, Downward J. PI3K pathway dependencies in endometrioid endometrial cancer cell lines. Clinical cancer research : an official journal of the American Association for Cancer Research 2013 Jul 1,;19(13):3533-3544.

19. Inaba et al. Antitumor activity of a combination of dual PI3K/mTOR inhibitor SAR245409 and selective MEK1/2 inhibitor pimasertib in endometrial carcinomas. Gynecologic Oncology 2015;138(2):323-331.

20. I Ray-coquard, L Favier, B Weber, C Roemer-becuwe, P Bougnoux, M Fabbro, et al. Everolimus as second- or third-line treatment of advanced endometrial cancer: ENDORAD, a phase II trial of GINECO. The British Journal of Cancer 2013 May 14;;108(9):1771-1777.

21. Matulonis et al. Phase II study of the PI3K inhibitor pilaralisib (SAR245408; XL147) in patients with advanced or recurrent endometrial carcinoma. Gynecologic Oncology 2014;136(2):246-253.

22. Josep Maria del Campo et al. A randomized phase II non-comparative study of PF-04691502 and gedatolisib (PF-05212384) in patients with recurrent endometrial cancer. Gynecologic Oncology 2016;142(1):62-69.

23. P-E Heudel, M Fabbro, C Roemer-Becuwe, M C Kaminsky, A Arnaud, F Joly, et al. Phase II study of the PI3K inhibitor BKM120 in patients with advanced or recurrent endometrial carcinoma: a stratified type I-type II study from the GINECO group. British journal of cancer 2017 Jan 1,;116(3):303-309.

24. Aslan O, Cremona M, Morgan C, Cheung LW, Mills GB, Hennessy BT. Preclinical evaluation and reverse phase protein Array-based profiling of PI3K and MEK inhibitors in endometrial carcinoma in vitro. BMC cancer 2018 Feb 9;18(1):168.

25. Suga et al. An inhibitory effect on cell proliferation by blockage of the MAPK/estrogen receptor/MDM2 signal pathway in gynecologic cancer. Gynecologic Oncology 2006;105(2):341-350.

26. Llobet et al. The multikinase inhibitor Sorafenib induces apoptosis and sensitises endometrial cancer cells to TRAIL by different mechanisms. European Journal of Cancer 2009;46(4):836-850.

27. Schrauwen et al. Dual blockade of PI3K/AKT/mTOR (NVP-BEZ235) and Ras/Raf/MEK (AZD6244) pathways synergistically inhibit growth of primary endometrioid endometrial carcinoma cultures, whereas NVP-BEZ235 reduces tumor growth in the corresponding xenograft models. Gynecologic Oncology 2015;138(1):165-173.

28. Coleman et al. A phase II evaluation of selumetinib (AZD6244, ARRY-142886), a selective MEK-1/2 inhibitor in the treatment of recurrent or persistent endometrial cancer: An NRG Oncology/Gynecologic Oncology Group study. Gynecologic Oncology 2015;138(1):30-35.

29. Wainberg Z, Alsina M, Soares H, Braña I, Britten C, Del Conte G, et al. A Multi-Arm Phase I Study of the $\mathrm{PI3K} / \mathrm{mTOR}$ Inhibitors PF-04691502 and Gedatolisib (PF-05212384) plus Irinotecan or the MEK Inhibitor PD-0325901 in Advanced Cancer. Targ Oncol 2017 Dec;12(6):775-785. 
30. Chou T, Talalay P. Analysis of combined drug effects: a new look at a very old problem. Trends in Pharmacological Sciences 1983;4:450-454.

31. Chen Y, Peubez C, Smith V, Xiong S, Kocsis-Fodor G, Kennedy B, et al. CUDC-907 blocks multiple prosurvival signals and abrogates microenvironment protection in CLL. Journal of Cellular and Molecular Medicine 2019;23(1):340-348.

32. U.S National Library of Medicine. , 2021.

33. Younes et al. Safety, tolerability, and preliminary activity of CUDC-907, a first-in-class, oral, dual inhibitor of HDAC and PI3K, in patients with relapsed or refractory lymphoma or multiple myeloma: an open-label, dose-escalation, phase 1 trial. Lancet Oncology, The 2016;17(5):622-631.

34. Oki Y, Kelly KR, Flinn I, Patel MR, Gharavi R, Ma A, et al. CUDC-907 in relapsed/refractory diffuse large B-cell lymphoma, including patients with MYC-alterations: results from an expanded phase I trial. Haematologica 2017 Nov;102(11):1923-1930.

35. Liu A, Zhang D, Yang X, Song Y. Estrogen receptor alpha activates MAPK signaling pathway to promote the development of endometrial cancer. Journal of cellular biochemistry 2019 Oct;120(10):17593-17601.

36. Chen Y, Germano S, Shelmani G, Kluczna D, Jayne S, Dyer M, et al. Paradoxical activation of alternative pro-survival pathways determines resistance to MEK inhibitors in chronic lymphocytic leukaemia. British journal of haematology 2017.

37. Balmanno K, Chell SD, Gillings AS, Hayat S, Cook SJ. Intrinsic resistance to the MEK $1 / 2$ inhibitor AZD6244 (ARRY-142886) is associated with weak ERK1/2 signalling and/or strong PI3K signalling in colorectal cancer cell lines. International Journal of Cancer 2009 Nov 15;125(10):2332-2341.

38. Yaeger R, Corcoran RB. Targeting Alterations in the RAF-MEK Pathway. Cancer discovery 2019 Mar;9(3):329-341.

39. Collins A, Miles GJ, Powley IR, Hew R, Pringle JH, MacFarlane M, et al. Development of a patientderived explant model for prediction of drug responses in endometrial cancer. Gynecologic oncology 2021 Feb;160(2):557-567.

40. Weinstein JN. Drug discovery: Cell lines battle cancer. Nature 2012 Mar 28;483(7391):544.

\section{Figures}


A Ishikawa HEC-1-A

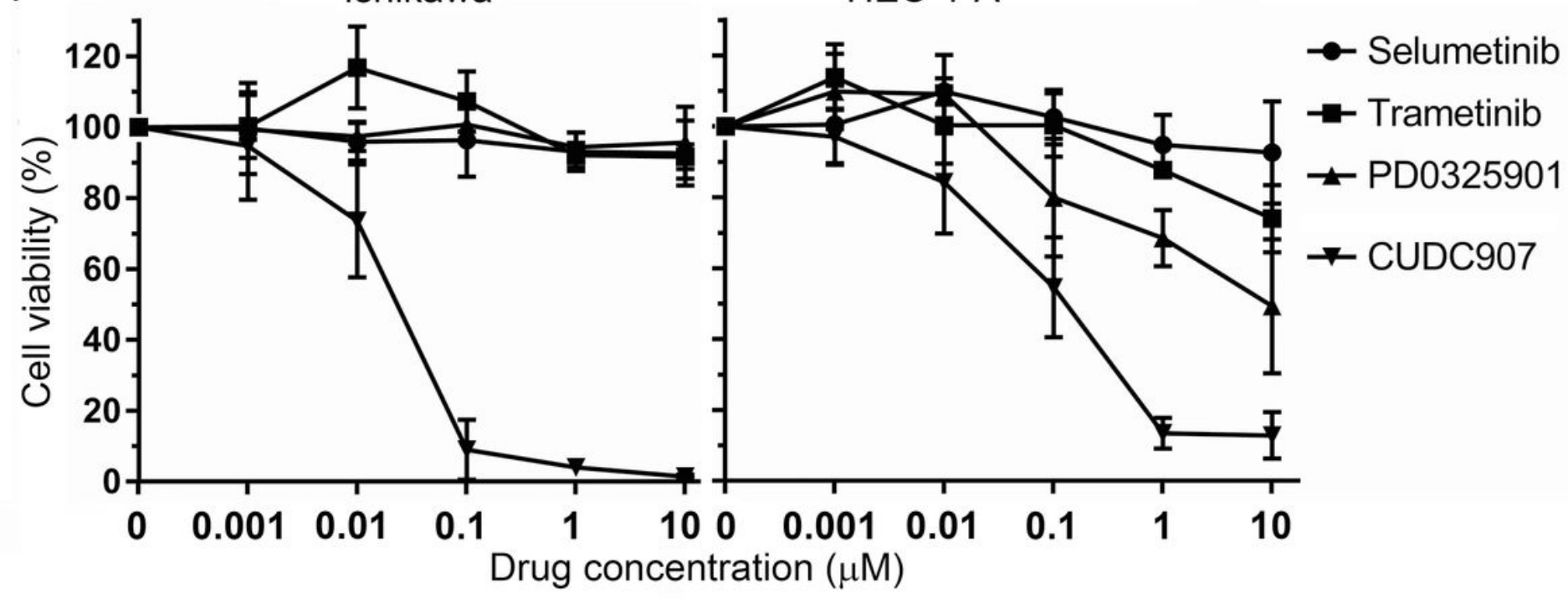

B

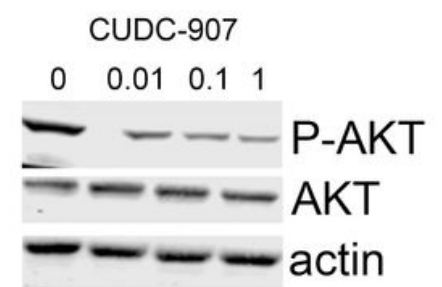

C Ishikawa HEC-1-A

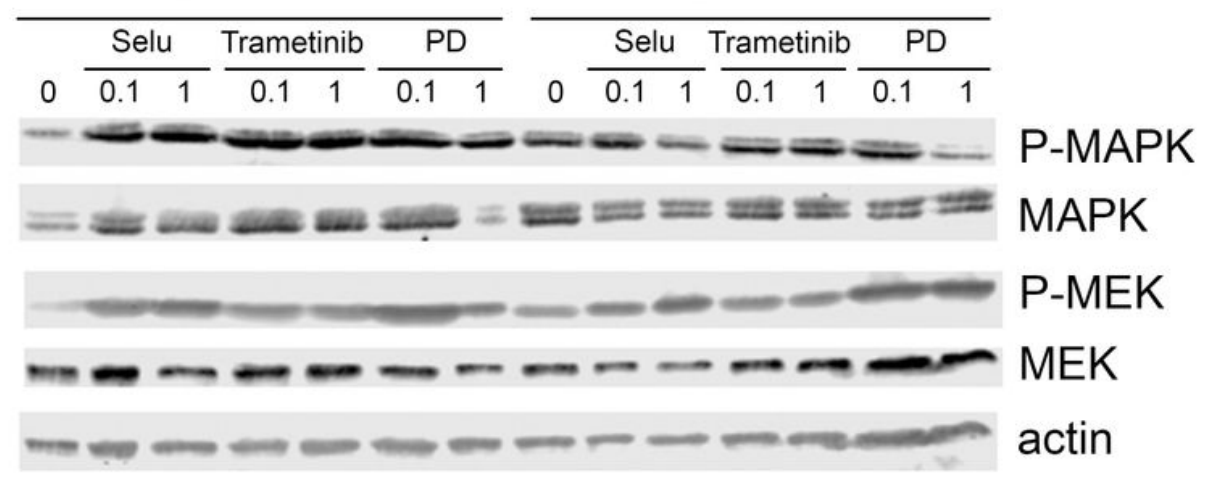

Figure 1

\section{Figure 1}

(A) Effect of treatment with serial concentrations of MEK inhibitors (Selumetinib, Trametinib and PD0325901) and the PI3K and HDAC inhibitor CUDC-907 on two endometrial cancer cell lines (Ishikawa and HEC-1-A). Viability was measured 48 hours after treatment using MTS assay. Experiments were performed in triplicates and repeated a minimum of three times. Error bars represent standard deviation of the mean. (B) Western blotting showing the expression of phospho-AKT and AKT in Ishikawa endometrial cancer cells treated with serial concentrations of CUDC-907 (PI3K and HDAC inhibitor) for 24 hours. (C) Western blotting showing the effect on MAPK and MEK phosphorylation of endometrial cancer cells (Ishikawa and HEC-1-A) after treating with serial concentrations of the MEK inhibitors Selumetinib, Trametinib and PD0325901 for 24 hours. 


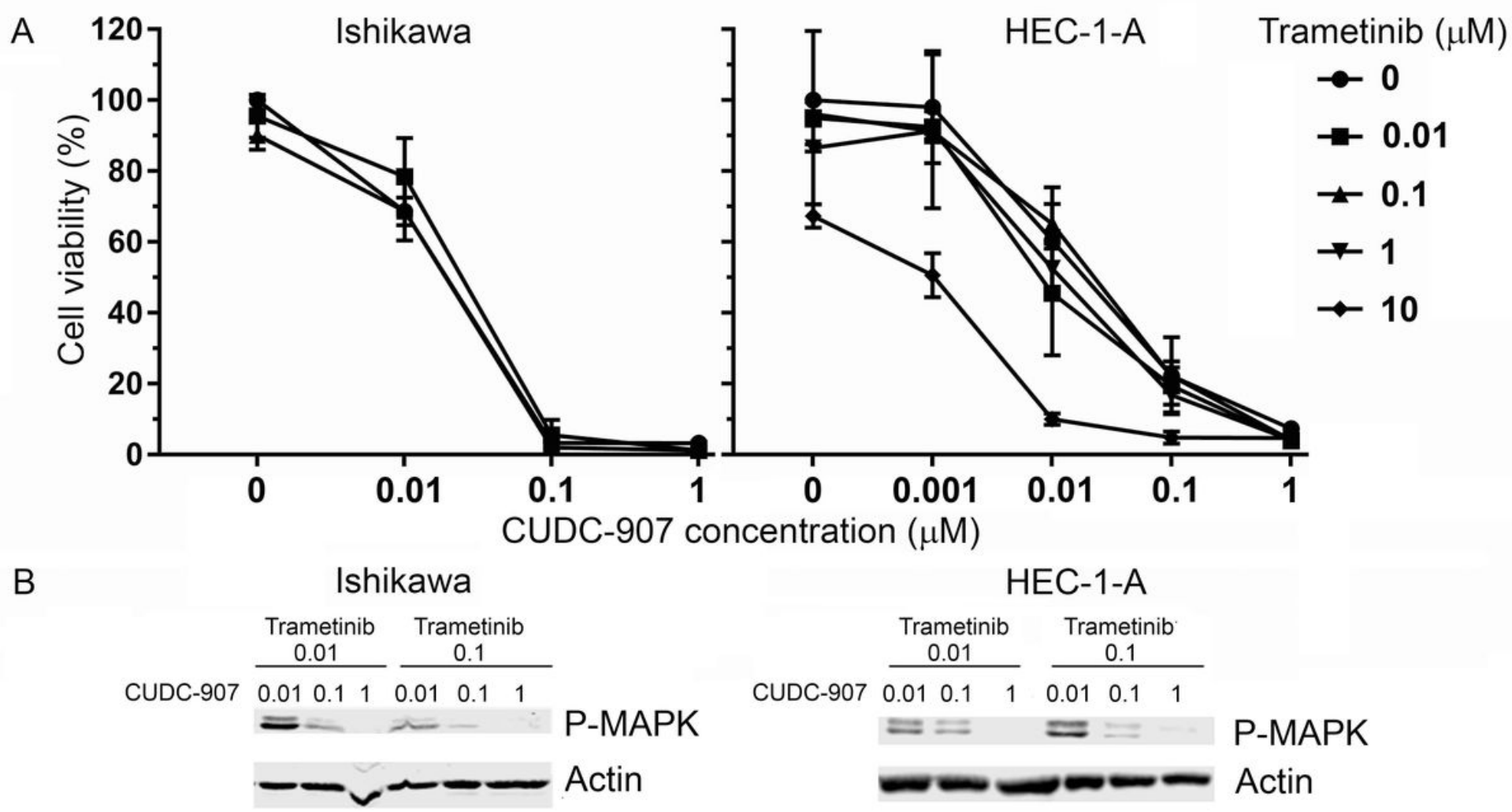

Figure 2

Figure 2

(A) Effect of Trametinib (MEK inhibitor) on viability of endometrial cancer cells. Viability was measured 48 hours after treatment using MTS assay. Experiments were performed in triplicates and repeated a minimum of three times. Error bars represent standard deviation of the mean. (B) Effect of dual MEK and PI3K inhibition (Trametinib and CUDC-907) on MAPK phosphorylation of endometrial cancer cells. Western blotting 24 hours after treatment with serial concentrations of the previously mentioned inhibitors. 
A

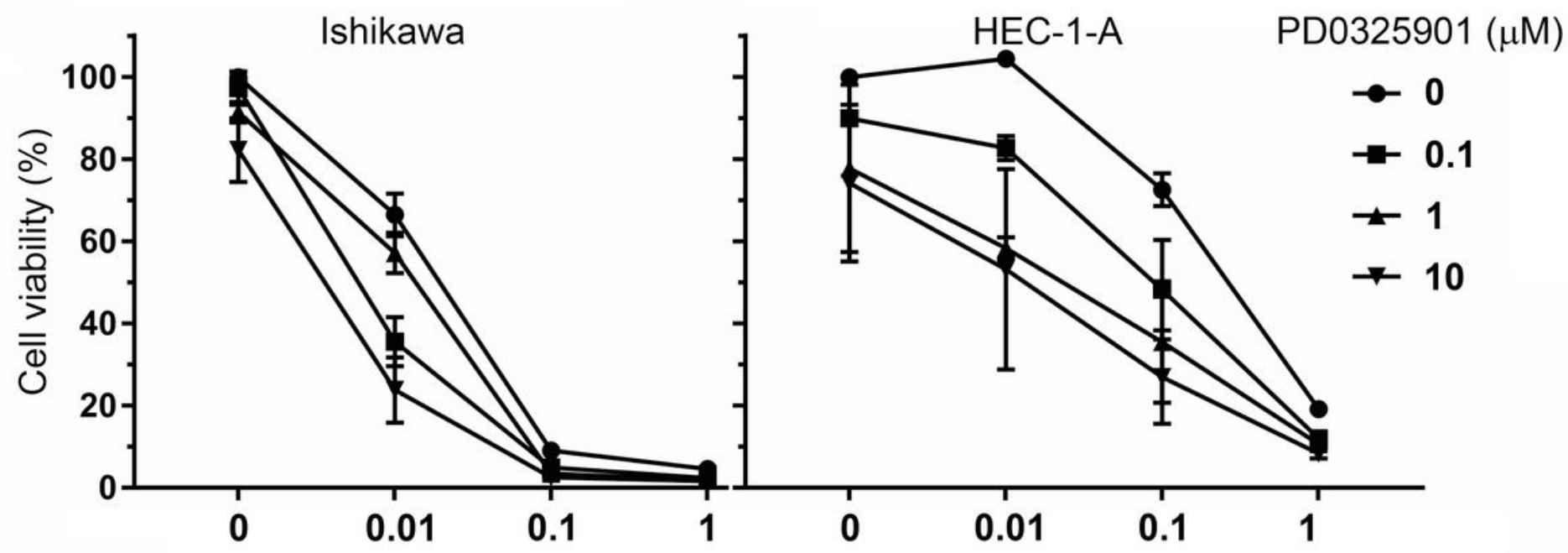

B

CUDC-907 concentration $(\mu \mathrm{M})$

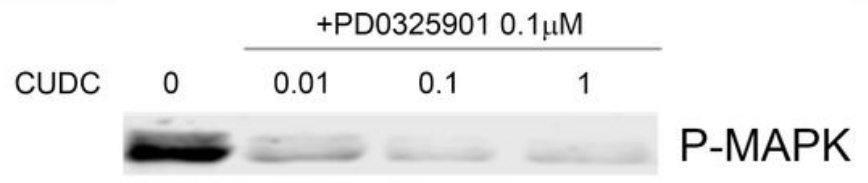

\section{P-MAPK}

Actin

Figure 3

Figure 3

(A) Effect of PI3K and HDAC inhibitor (CUDC-907) combined with MEK inhibitor (PD0325901) in viability of endometrial cancer cells after 48 hours of treatment, using MTS assay. Experiments were performed in triplicates and repeated a minimum of three times. Error bars represent standard deviation of the mean. (B) Effect of dual MEK and PI3K inhibition (PD0325901 and CUDC-907) on MAPK phosphorylation of Ishikawa endometrial cancer cells. Western blotting 24 hours after treatment with serial concentrations of the previously mentioned inhibitors.

\section{Supplementary Files}

This is a list of supplementary files associated with this preprint. Click to download.

- Originalblotscelllinepaper.pptx 